\title{
Clinical evaluation of a rapid diagnostic test kit for detection of canine coronavirus
}

\author{
Seung-Jae Yoon, Kyoung-Won Seo, Kun-Ho Song* \\ College of Veterinary Medicine, Chungnam National University, Daejeon 34134, South Korea
}

(Received: November 27, 2017; Revised: March 12, 2018; Accepted: March 20, 2018)

\begin{abstract}
Canine coronavirus is a single-stranded RNA virus that causes enteritis in dogs of any age. Coronaviral enteritis is seldom definitively diagnosed, since it is usually much less severe than many other types of enteritis and is self-limiting. Conventional diagnostics for the canine coronaviral enteritis such as polymerase chain reaction (PCR), virus isolation, and electron microscopic examination are inappropriate for small animal clinics due to the complicated experimental processes involved. Therefore, a commercially available lateral flow test kit based on chromatographic immunoassay techniques was tested to evaluate its performance as a first-line diagnostic test kit that could be used in clinics. The coronavirus antigen test kit detected canine coronavirus-infected dogs with $93.1 \%$ sensitivity and $97.5 \%$ specificity. The detection limit of the test kit was between $1.97 \times 10^{4} / \mathrm{mL}$ and $9.85 \times 10^{3} / \mathrm{mL}$ for samples with a 2fold serial dilution from $1.25 \times 10^{6} \mathrm{TCID}_{50}\left(\mathrm{TCID}_{50}, 50 \%\right.$ tissue culture infectious dose). Additionally, the test kit had no cross-reactivity with canine parvovirus, distemper virus, or Escherichia coli. Overall, the commercially available test kit showed good diagnostic performance in a clinical setting, with results similar to those from PCR, confirming their potential for convenient and accurate use in small animal clinics.
\end{abstract}

Keywords: antigen test kit, coronavirus, dogs, polymerase chain reaction

\section{Introduction}

The canine coronavirus (CCV) is a single-stranded RNA virus that causes mild to severe enteritis in dogs of all ages $[1,2]$. Canine coronaviral enteritis occurs when the coronavirus invades and destroys mature cells on the intestinal villi, resulting in a reduction of absorptive surface area and malabsorption [7, 10]. Clinical signs include anorexia, depression, vomiting, and diarrhea. Most dogs recover in seven to ten days, and the mortality rate is low. However, young dogs may die due to dehydration or electrolyte abnormalities if they are not treated. Additionally, co-infection with adenovirus, parvovirus or distemper virus can increase mortality [1, 3-7, 11].

Coronaviral enteritis is seldom definitively diagnosed, since it is usually much less severe than many other enteritis and is self-limiting. Even in severe cases, infected dogs are responsive to supportive care. Nonetheless, diagnostic tests are indicated to differentiate coronavirus infection from other diseases that initially mimic coronavirus infection in order to treat the patient and predict prognosis properly based on definitive diagnosis.

Coronavirus can be isolated in a number of cell lines, but the procedure is time-consuming and unreliable. Polymerase chain reaction (PCR) is also available for the detection of coronavirus in canine feces, but it requires special equipment, skilled operators, and a well-organized laboratory.

For these reasons, a commercial rapid canine coronavirus antigen detection test kit, a lateral flow test kit using chromatographic immunoassay techniques. The purpose of this study is to evaluate the performance of the commercial rapid test kit as a first-line diagnostic test kit that can be used in veterinary clinics.

\section{Materials and Methods}

\section{Specimen}

This study was performed using canine fecal samples collected from April 2014 to March 2015. Out of 179 fecal samples, 58 were positive for CCV and 121 were negative. Over ten different animal hospitals in South Korea sent fecal samples from dogs suspected of having CCV infection and requested PCR assays from the laboratory during the study period.

\section{Lateral flow test kit}

The Antigen Rapid CCV Ag Test kit (BioNote, Korea)

*Corresponding author

Tel: +82-42-821-6789, Fax: +82-42-821-6789

E-mail: songkh@cnu.ac.kr 
A

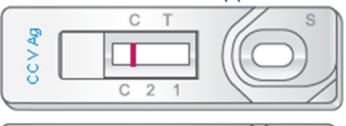

B
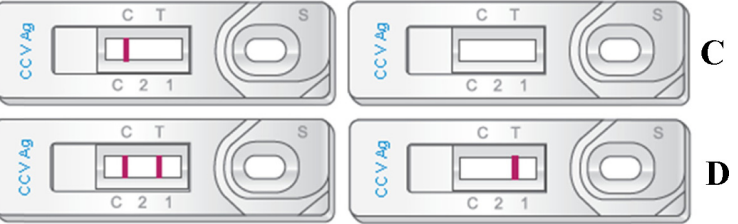

Fig. 1. Illustrations of the Antigen Rapid CCV Ag Test Kit results. (A) Negative result. (B) Positive result. (C and D) Invalid result.

(Fig. 1) was used according to the manufacturer's instructions. In brief, fecal samples were thawed and diluted using assay diluents for CCV until they were fully dissolved (approximately $10 \mathrm{sec}$ ). The samples were then incubated at room temperature for 1 minute to allow the large particles to settle. Using a disposable dropper, four drops of the supernatant from the diluted sample were added to the sample hole for each test device. Test results were read after 10 minutes. The sample was regarded as positive if both the test (" $T$ ") line and control ("C") line were present within the result window. The sample was regarded as negative if only the control ("C") line appeared in the result window, and as invalid if the control ("C") line did not appear.

\section{Nested PCR}

Viral RNA extraction: In brief, $100 \mu \mathrm{L}$ of fecal sample was transferred into RNase-free water containing a microcentrifuge tube (Eppendorf, Germany). After adding $900 \mu \mathrm{L}$ of Trizol, the sample was mixed gently and left at room temperature for 5 minutes. Next, $200 \mu \mathrm{L}$ of chloroform was added and mixed thoroughly. After 3 minutes, the sample was centrifuged at 43,500 $\times \mathrm{g}$ (Hanil Science, Korea) for $10 \mathrm{~min}$, and the supernatant was transferred to a new microcentrifuge tube (Eppendorf). After the addition of $500 \mu \mathrm{L}$ of iso-ethanol, the solution was mixed vigorously, then left at room temperature for $10 \mathrm{~min}$. After centrifuging (Hanil Science) the sample at $43,500 \times \mathrm{g}$ for $10 \mathrm{~min}$, the supernatant was removed, and the pellet was washed with $1 \mathrm{~mL}$ of $75 \%$ ethanol. The sample was centrifuged (Hanil Science) again at $43,500 \times \mathrm{g}$ for $10 \mathrm{~min}$, and the supernatant was removed. The remaining pellet was dried on a clean bench for $15 \mathrm{~min}$ and dissolved with $10 \mu \mathrm{L}$ of diethyl pyrocarbonate (DEPC) water (Sigma-Aldrich, USA).

Primers for reverse transcription PCR (RT-PCR) and Nested PCR: Nested PCR uses two primer sets. The first primer sets used in this study were primer CCV gP1 F and primer $\mathrm{CCV}$ gP1 $\mathrm{R}$ The second primer sets were primer $\mathrm{CCV}$ gP2 F and primer CCV gP2 R (Table 1).

RT-PCR: One microliter of extracted RNA was mixed with $2 \mu \mathrm{L}$ of 10 -fold-diluted CCVR1 primer, and the mixture was left at $70^{\circ} \mathrm{C}$ for $5 \mathrm{~min}$. Next, $15 \mu \mathrm{L}$ of DEPC water and $2 \mu \mathrm{L}$ of 10 -fold-diluted CCVF1 primer were added to the Bioneer RT Premix (AccuPower RT PreMix; Bioneer, Korea). Both solutions were mixed together, and RT-PCR was performed. The temperature condition was set as the following:
Table 1. Primers for reverse transcription polymerase chain reaction (RT-PCR) and nested polymerase chain reaction (nested-PCR)

\begin{tabular}{cc}
\hline \hline Accession No. & \multicolumn{1}{c}{ Sequence $\left(5^{\prime}-3^{\prime}\right)$} \\
\hline CCV gP1 F & TAATGTGACACAAYTGCCTGGCAATG \\
CCV gP1 R & CTGTAGAAACTYGACTCACTCACTG \\
CCV gP2 F & GTACTGGCAATGCAMGWGGTAAACC \\
CCV gP2 R & ACRTTGGTNGCATAGCCAGTGCA \\
\hline
\end{tabular}

$\mathrm{F}$, forward; R, reverse.

$\mathbf{A}$

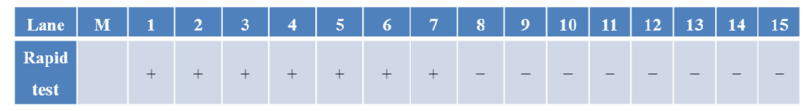

B

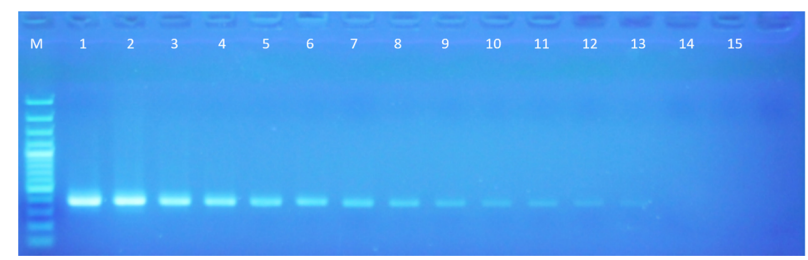

Fig. 2. Detection limit test result for the Antigen rapid $\mathrm{CCV} \mathrm{Ag}$ Test Kit (A) and nested PCR (B). M, marker; Lane 1, $1.25 \times 10^{6}$ $\mathrm{TCID}_{50} / \mathrm{mL}$ of CCV positive sample; Lanes 2-14, a two-fold series of dilutions $\left(6.25 \times 10^{5}-1.55 \times 10^{2} \mathrm{TCID}_{50} / \mathrm{mL}\right)$; Lane 15 , negative control.

initial denaturation at $94^{\circ} \mathrm{C}$ for 10 minutes, followed by 33 cycles of denaturation at $94^{\circ} \mathrm{C}$ for $25 \mathrm{sec}$, annealing at $58^{\circ} \mathrm{C}$ for $30 \mathrm{sec}$, extension at $72^{\circ} \mathrm{C}$ for $2 \mathrm{~min}$, and elongation at $72^{\circ} \mathrm{C}$ for $5 \mathrm{~min}$.

Nested PCR: Nested PCR was performed on a BIORAD DNA Engine PTC 0200 (USA) according to the method by Naylor et al. [12]. One microliter of the amplified RT-PCR product, which was diluted 10-fold with DEPC water, was used as a template. It was mixed with the reaction solution including dNTP mix (each $10 \mathrm{mM}$ ), 10× PCR buffer, $5 \times \mathrm{Q}$ solution, Tag polymerase, and CCVF2 and R2 primers. The cycling condition for nested PCR was the same as that for RT-PCR. The products of the nested PCR reactions were analyzed with agarose gel electrophoresis to assess the presence of the amplified bands at $514 \mathrm{bp}$ (Fig. 2).

\section{Diagnostic sensitivity, specificity, and accuracy}

All of the collected 179 samples were tested with the rapid kit. The diagnostic sensitivity, specificity, and accuracy of the kit were calculated as shown in Table 2 .

\section{Detection limit}

Samples of the CCV DS 2 strain $\left(1.25 \times 10^{6} \mathrm{TCID}_{50} / \mathrm{mL}\right.$; $\mathrm{TCID}_{50}, 50 \%$ tissue culture infectious dose). and its 2-fold serial dilutions were prepared in order to evaluate the limit of detection for the rapid test kit compared with that of nested 
Table 2. Formula for calculating diagnostic sensitivity, specificity, and accuracy

\begin{tabular}{llccc}
\hline \hline & & Gold standard method (nested PCR) & Total \\
\cline { 3 - 4 } & & Positive & Negative & \\
\hline \multirow{2}{*}{ Antigen Rapid CCV Ag Test Kit } & Positive & $\mathrm{a}$ & $\mathrm{b}$ & $\mathrm{a}+\mathrm{b}$ \\
& Negative & $\mathrm{c}$ & $\mathrm{d}$ & $\mathrm{c}+\mathrm{d}$ \\
& Total & $\mathrm{a}+\mathrm{c}$ & $\mathrm{b}+\mathrm{d}$ & $\mathrm{a}+\mathrm{b}+\mathrm{c}+\mathrm{d}$ \\
\hline
\end{tabular}

Diagnostic sensitivity $(\%)=a /(a+c) \times 100 ;$ Diagnostic specificity $(\%)=d /(b+d) \times 100 ;$ Accuracy $(\%)=(a+d) /(a+b+c+d) \times 100$.

Table 3. Sensitivity and specificity test results for nested PCR and the Antigen Rapid CCV Ag Test Kit

\begin{tabular}{llccr}
\hline \hline & & \multicolumn{2}{c}{ Nested PCR } & Total \\
\cline { 3 - 4 } & & Positive & Negative & 57 \\
Antigen Rapid CCV Ag Test Kit & Positive & 54 & 3 & 122 \\
& Negative & 4 & 118 & 121 \\
& Total & 58 & 121 & 179 \\
\hline
\end{tabular}

Sensitivity $(\%)=54 / 58 \times 100=93.1 ;$ Specificity $(\%)=118 / 121 \times 100=97.5 ;$ Accuracy $(\%)=172 / 179 \times 100=96.1$

Table 4. The detection limit of the commercial rapid CCV Ag test kit compared to nested PCR

\begin{tabular}{cccccc}
\hline \hline Titer $\left(\mathrm{TCID}_{50} / \mathrm{mL}\right)$ & Rapid kit & Nested PCR & Titer $\left(\mathrm{TCID}_{50} / \mathrm{mL}\right)$ & Rapid kit & Nested PCR \\
\hline $1.25 \times 10^{6}$ & Positive & Detected & $9.85 \times 10^{3}$ & Negative & Detected \\
$6.25 \times 10^{5}$ & Positive & Detected & $4.93 \times 10^{3}$ & Negative & Detected \\
$3.13 \times 10^{5}$ & Positive & Detected & $2.47 \times 10^{3}$ & Negative & Detected \\
$1.57 \times 10^{5}$ & Positive & Detected & $1.24 \times 10^{3}$ & Negative & Detected \\
$7.85 \times 10^{4}$ & Positive & Detected & $6.20 \times 10^{2}$ & Negative & Detected \\
$3.93 \times 10^{4}$ & Positive & Detected & $3.10 \times 10^{2}$ & Negative & Detected \\
$1.97 \times 10^{4}$ & Positive & Detected & $1.55 \times 10^{2}$ & Negative & Not detected \\
\hline
\end{tabular}

PCR. Assay diluents included with the Antigen rapid CCV Ag kit were prepared as the negative controls. Fifteen concentrations of the samples were assayed using the rapid test kit and nested PCR.

\section{Cross-reactivity}

Canine distemper virus, canine parvovirus, porcine transmissible gastroenteritis virus (TGEV), and Escherichia coli (E. coli) were prepared to evaluate the cross-reactivity of the rapid test kit. The titers for these canine-related viruses were between $10^{5}$ and $10^{6} \mathrm{TCID}_{50} / \mathrm{mL}$, and the concentration of $E$. coli was $10^{8} \mathrm{CFU} / \mathrm{mL}$ (CFU, colony-forming unit).

\section{Results}

\section{Sensitivity and specificity}

To evaluate the accuracy of the rapid test kit, serum samples were obtained from dogs naturally infected with CCV. Fifty-four of the 58 PCR-positive samples had positive results when tested using the rapid test kit and three out of 121 PCR-negative samples had positive results when tested using the rapid test kit. Accordingly, with the samples evaluated in this study, the sensitivity and specificity of the rapid test kit were $93.1 \%$ and $97.5 \%$, respectively (Table 3 ).
Table 5. Cross reactivity of commercial CCV rapid test kit

\begin{tabular}{lc}
\hline \hline & Cross-reactivity \\
\hline Canine parvovirus & No \\
Canine distemper virus & No \\
Porcine transmissible gastroenteritis virus & Yes \\
Escherichia coli & No \\
\hline
\end{tabular}

\section{Detection limit}

The performance of the Antigen rapid CCV Ag kit was first assessed in-house with field samples, and was compared with the results of the PCR test conducted in parallel. The detection limit for the rapid test kit was $1.97 \times 10^{4} / \mathrm{mL}$ $-9.85 \times 10^{3} / \mathrm{mL}$ irrespective of the serotype when tested with the samples serially diluted 2-fold from $1.25 \times 10^{6} \mathrm{TCID}_{50} / \mathrm{mL}$ (Table 4).

\section{Cross-reactivity}

Fifty-eight dogs were PCR-positive for CCV, and none of the dogs were PCR-positive for canine parvovirus, canine distemper virus, and E. coli. However, the rapid test kit showed cross-reactivity with TGEV, which is included in the coronaviral group (Table 5). Additionally, 34 dogs were found 
to be co-infected with canine parvovirus and 16 dogs were co-infected with canine Giardiasis.

\section{Discussion}

In this study, we evaluated the performance of the commercial rapid test kit for detecting the $\mathrm{CCV}$ antigen. The PCR experiment was selected as the gold standard test because it is commonly used by various veterinary diagnostic laboratories and has been utilized in many large-scale tests.

Fifty-four of 58 PCR-positive samples were recognized as positive for CCV by the rapid test kit, while 118 of 121 PCRnegative samples were recognized as negative. The sensitivity and specificity of the Antigen rapid CCV Ag test kit for CCV detection were $93.1 \%$ and $97.5 \%$, respectively, compared with those of nested PCR. False negative results can occur when a positive sample contains a concentration of the $\mathrm{CCV}$ antigen that is lower than the detection limit for the rapid test kit. Also, fecal samples containing complexed antigens fail to react with the test antibody, resulting in false negative results. False positive results can occur due to nonspecific binding reactions, and have been linked to uncertain substances in the fecal sample. Some substances in the sample can bind to the trapping antibody (colloidal gold-conjugated labeled antibody coating the conjugated pad), forming a complex that can be easily detected by the capture antibody coated on the test-line of the strip, which results in a false positive [9]. Because of these false negative and false positive results, many authors think that the rapid test kit alone has limited diagnostic value in clinics. However, false positives occur in only a small percentage of cases and therefore as a first-line diagnostic test kit, the performance of the rapid test kit is satisfactory.

The detection limit for the Antigen rapid CCV Ag kit was confirmed to be $1.97 \times 10^{4} / \mathrm{mL}-9.85 \times 10^{3} / \mathrm{mL}$. A 2-fold difference in virus concentration was sufficient to determine an accurate detection cutoff for rapid detection of CCV. The commercial rapid test kit is considered satisfactory for use in the detection of $\mathrm{CCV}$ antigen in small animal clinics. It should be noted that the detection limit determined here is an estimate for field samples. Further investigation is required to accurately determine the detection limit of the assay on cultured viruses.

$\mathrm{CCV}$ is a virus in the Coronaviridae family, which can be divided into three antigenic groups [13]. Group 1 coronavirus includes CCV, TGEV, feline coronavirus, and the human coronavirus $229-\mathrm{E}$. These viruses can serologically cross-react with each other [8, 14], and genomic analysis shows close relationships between them [14]. The Antigen rapid CCV Ag kit did not show cross-reactions with other viruses except for TGEV. Therefore, it is recommended to detect CCV antigen using immunological assays to prevent cross-reactions with other group 1 coronaviruses.

The Antigen Rapid CCV Ag kit showed good diagnostic performance in a clinical setting, with results similar to those from PCR experiments. There were no significant differences in the test results of the commercial kit and PCR analysis. A lower number of positive results $(n=54)$ was obtained from the former compared to the latter $(n=58)$, resulting in a higher number of false negatives. However, the PCR assay depends heavily on an experienced technician performing the experiments and is not always available, whereas the commercial rapid test kit is a very easy-to-use kit. This technique is very simple to perform and to interpret enabling efficient use in animal clinics.

\section{Acknowledgments}

This study was supported by the research fund of BIONOTE (South Korea).

\section{References}

1. Appel MJ. Canine coronavirus. In: Appel MJ (ed.). Virus Infections of Carnivores. Elsevier Science, Amsterdam, 1987.

2. Binn LN, Lazar EC, Keenan KP, Huxsoll DL, Marchwicki RH, Strano AJ. Recovery and characterization of a coronavirus from military dogs with diarrhea. Proc Ann Meet U S Anim Health Assoc 1974, 359-366.

3. Decaro N, Camero M, Greco G, Zizzo N, Tinelli A, Campolo M, Pratelli A, Buonavoglia C. Canine distemper and related diseases: report of a severe outbreak in a kennel. New Microbiol 2004, 27, 177-181.

4. Decaro N, Campolo M, Elia G, Buonavoglia D, Colaianni ML, Lorusso A, Mari V, Buonavoglia C. Infectious canine hepatitis: an "old" disease reemerging in Italy. Res Vet Sci 2007, 83, 269-273.

5. Decaro N, Desario C, Elia G, Campolo M, Lorusso A, Mari V, Martella V, Buonavoglia C. Occurrence of severe gastroenteritis in pups after canine parvovirus vaccine administration: a clinical and laboratory diagnostic dilemma. Vaccine 2007, 25, 1161-1166.

6. Decaro N, Martella V, Desario C, Bellacicco AL, Camero M, Manna L, D'Aloja D, Buonavoglia C. First detection of canine parvovirus type $2 \mathrm{c}$ in pups with haemorrhagic enteritis in Spain. J Vet Med B Infect Dis Vet Public Health 2006, 53, 468-472.

7. Greene CE. Canine coronaviral enteritis. In: Greene CE (ed). Infectious Diseases of the Dog and Cat. pp. 281-283. WB Saunders, Philadelphia, 1990.

8. Horzinek MC, Lutz H, Pedersen NC. Antigenic relationships among homologous structural polypeptides of porcine, feline, and canine coronaviruses. Infect Immun 1982, 37, 1148-1155.

9. Iqbal J, Sher A, Rab A. Plasmodium falciparum histidinerich protein 2-based immunocapture diagnostic assay for malaria: cross-reactivity with rheumatoid factors. J Clin Microbiol 2000, 38, 1184-1186.

10. Keenan KP, Jervis HR, Marchwicki RH, Binn LN. Intestinal infection of neonatal dogs with canine coronavirus 1-71: studies by virologic, histologic, histochemical, and immunofluorescent techniques. Am J Vet Res 1976, 37, 247256.

11. Martin HD, Zeidner NS. Concomitant cryptosporidia, coronavirus and parvovirus infection in a raccoon (Procyon 
lotor). J Wild Dis 1992, 28, 113-115.

12. Naylor MJ, Harrison GA, Monckton RP, McOrist S, Lehrbach PR, Deane EM. Identification of canine coronavirus strains from feces by $\mathrm{S}$ gene nested PCR and molecular characterization of a new Australian isolate. J Clin Microbiol 2001, 39, 1036-1041.
13. Olsen CW. A review of feline infectious peritonitis virus: molecular biology, immunopathogenesis, clinical aspects, and vaccination. Vet Microbiol 1993, 36, 1-37.

14. Pedersen NC, Ward J, Mengeling WL. Antigenic relationship of the feline infectious peritonitis virus to coronaviruses of other species. Arch Virol 1978, 58, 45-53. 